\title{
Comparison between UNICON Index and Bispectral Index using Population Pharmacodynamic Analysis during Sevoflurane Anesthesia
}

\author{
Seongwook Jeong, S. Lee, T. Pyeon, H. Bae, E. Jang \\ Department of Anesthesiology and Pain Medicine, \\ Chonnam National University Medical School, South Korea
}

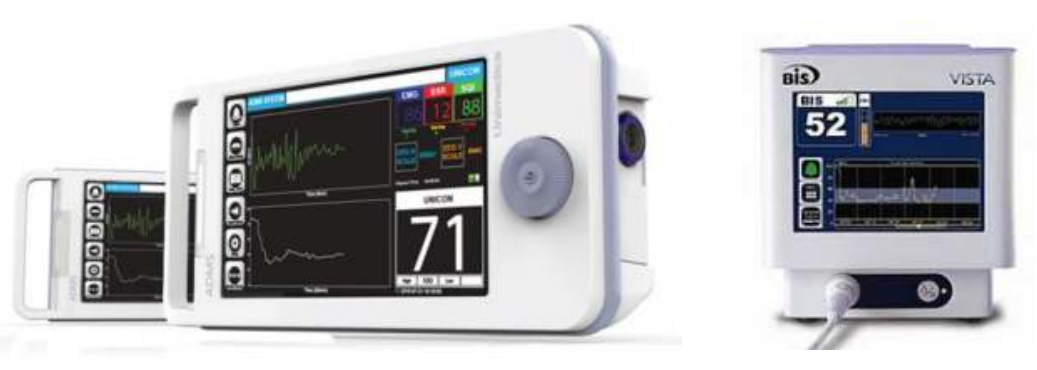

\section{Background and Goal of Study:}

ADMS (Anesthetic Depth Monitor for Sedation, Unimedics Co., Ltd., Seoul, Korea) is a recently introduced anesthetic depth measurement system, which displays the patient's arousal state as the UNICON Index. We performed pharmacodynamic (PD) analysis to compare the performance of the UNICON index to the bispectral index (BIS) during general anesthesia.

\section{Materials and Methods:}

We observed 30 adult patients scheduled for laparoscopic gastrectomy with a combined epidural-sevoflurane general anesthesia. During a phase of constant surgical stimulation, endtidal sevoflurane concentrations were varied between 1 - 4 vol\%. This procedure was repeated twice (15 min interval). End-tidal sevoflurane concentration, BIS, and UNICON index were recorded simultaneously.

Population PD models based on double-sigmoid curves were developed by NONMEM VII software (Icon Development Solutions, LLC, Ellicott City, USA).

$E=E_{\text {max }}+\left(E_{0}-E_{\text {plat }}\right) /\left(1+\frac{C e^{\gamma 1}}{C e 50_{1}^{\gamma 1}}\right)+\left(E_{\text {plat }}-E_{\text {max }}\right) /\left(1+\frac{C e^{\gamma 2}}{C e 50_{2}^{\gamma 2}}\right)$

- $E=$ electroencephalographic effect (BIS or UNICON)

- $E_{0}=$ baseline value for $E(B I S=100, U N I C O N=99$ fixed $)$

- $E_{\max }=$ maximum value for $E$ (BIS, UNICON=0 fixed)

- $E$ plat $=$ plateau that is the maximum effect of the $1^{\text {st }}$ sigmoidal curve and simultaneously the minimum effect of the $2^{\text {nd }}$ sigmoidal curve
- $\mathrm{Ce} 501, \mathrm{Ce} 502=$ the effect-site concentration associated with $50 \%$ decrease from Eo to Eplat and from Eplat to Emax

- Gam1, Gam2 = the steepness of the concentration-response relation curve for $1^{\text {st }}$ and $2^{\text {nd }}$ sigmoid curves

- $\mathrm{Ke} 0=$ rate constant determining the efflux from the effect-site

\section{Results and Discussion:}

A significant $(P<0.001)$ correlation between BIS and UNICON index was found for the experimental periods $\left(r^{2}=0.60\right.$, Pearson correlation coefficient).

The individual $\mathrm{E}_{\text {plat }}, \mathrm{Ce} 5 \mathrm{O}_{2}$, and ke0 for UNICON did not differ from those of BIS. The $\mathrm{Ce} 50_{1}$ was significantly smaller $(\mathrm{p}$ $<0.001$ ) for UNICON than for BIS, however, its clinical importance may be limited.

Table. Comparison of PD parameters between BIS and UNICON

\begin{tabular}{ccc}
\hline & BIS & UNICON \\
\hline Eplat & $38.6 \pm 5.0$ & $37.9 \pm 3.8$ \\
Ce501 & $0.69 \pm 0.25$ & $0.43 \pm 0.27^{*}$ \\
Ce502 & $3.87 \pm 1.50$ & $3.42 \pm 0.96$ \\
Gam1 & 3.02 & 4.44 \\
Gam2 & 13.3 & 17.54 \\
Ke0 & $0.25 \pm 0.13$ & $0.29 \pm 0.45$ \\
\hline
\end{tabular}

\section{Conclusion:}

Population pharmacodynamic models using double-sigmoid curves adequately described the responses of BIS and UNICON index to sevoflurane anesthesia, and most pharmacodynamic parameters for the BIS and UNICON index were similar.

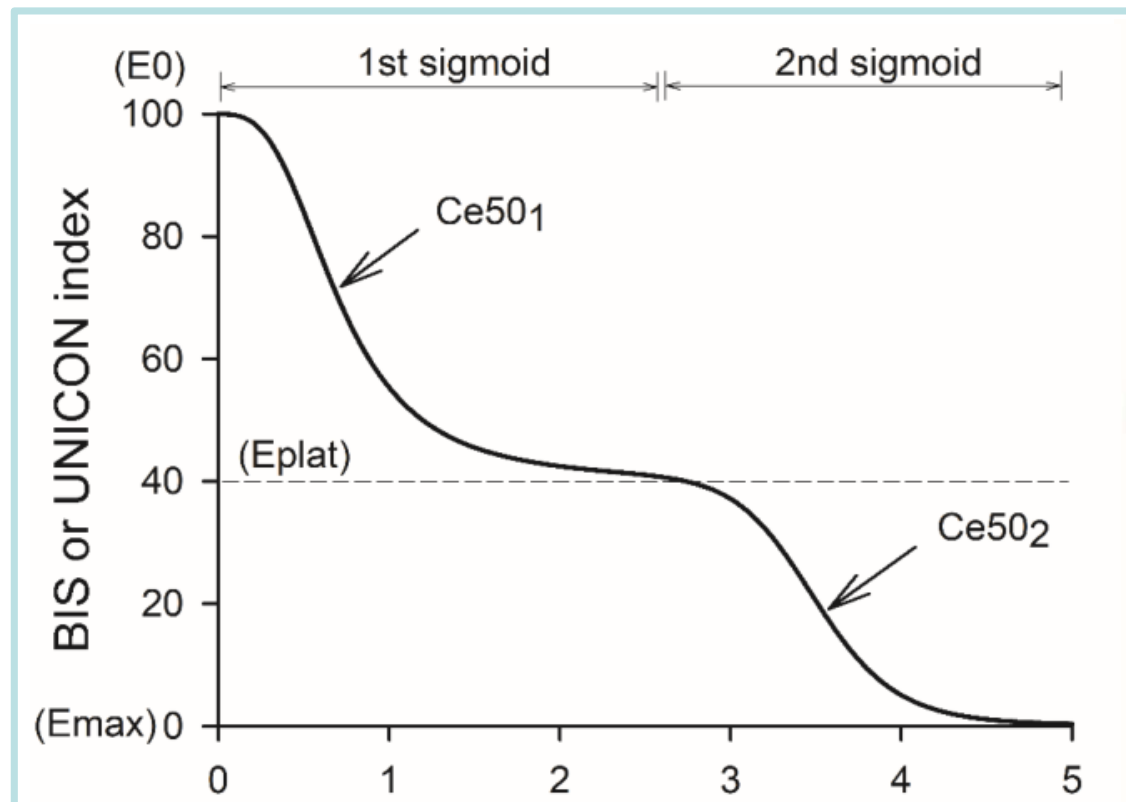

Sevoflurane effect-site concentration (vol\%)

Fig 1. Schematic presentation of the double-sigmoid model

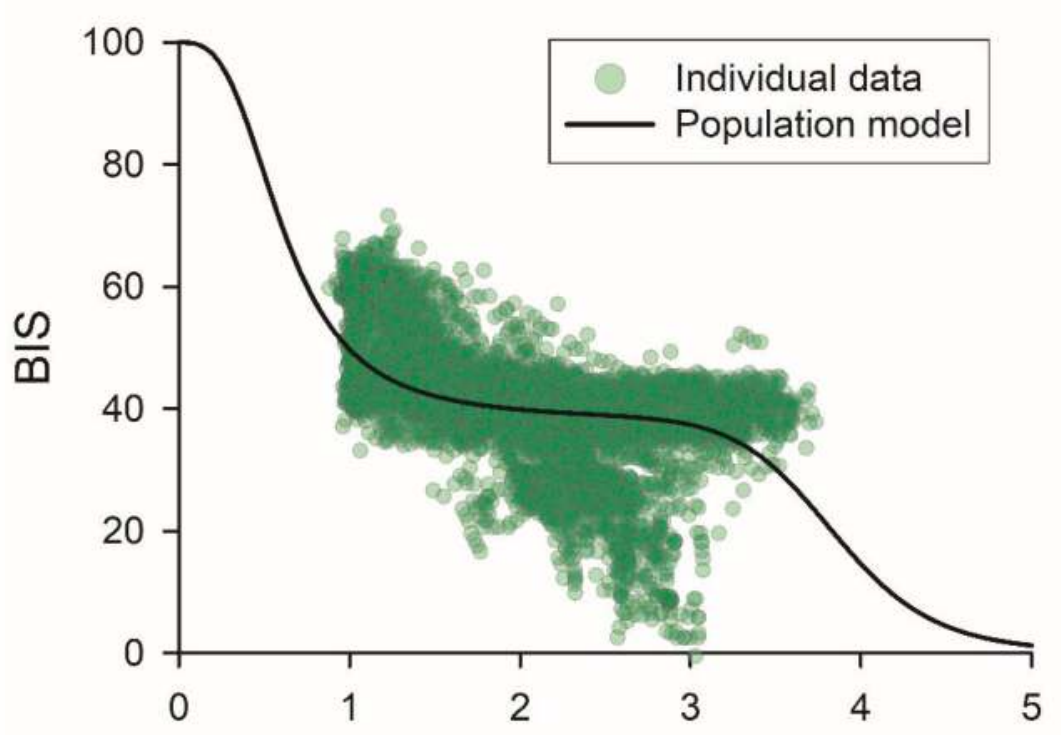

Sevoflurane effect-site concentration (vol\%)

Fig 2. Response of BIS to changes in sevoflurane effect-site concentrations

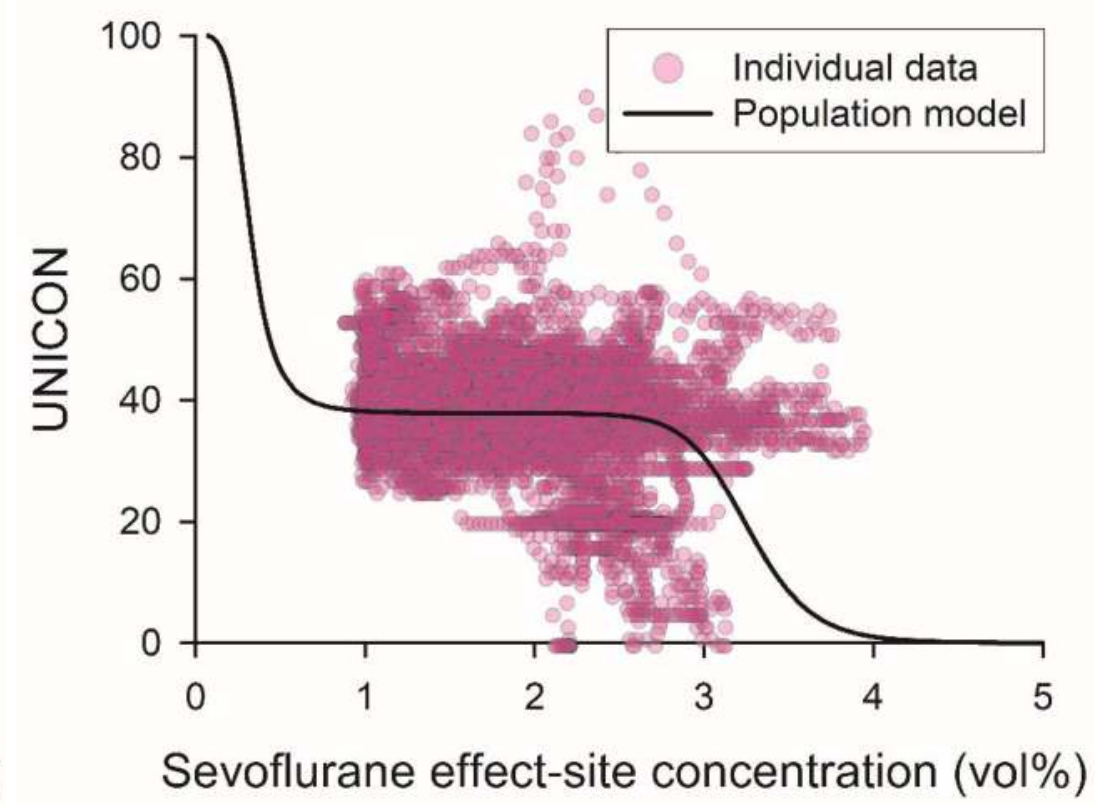

Fig 3. Response of UNICON to changes in sevoflurane effect-site concentrations 